\title{
ON THE STABLE CONTAINMENT OF TWO SETS
}

\author{
MIGUEL A. GOBERNA* AND VIRGINIA N. VERA DE SERIO ${ }^{\dagger}$
}

\begin{abstract}
This paper studies the stability of the set containment problem. Given two non-empty sets in the Euclidean space which are the solution sets of two systems of (possibly infinite) inequalities, the Farkas type results allow to decide whether one of the two sets is contained or not in the other one (which constitutes the so-called containment problem). In those situations where the data (i.e., the constraints) can be affected by some kind of perturbations, the problem consists of determining whether the relative position of the two sets is preserved by sufficiently small perturbations or not. This paper deals with this stability problem as a particular case of the maintaining of the relative position of the images of two set-valued mappings; first for general set-valued mappings and second for solution sets mappings of convex and linear systems. Thus the results in this paper could be useful in the postoptimal analysis of optimization problems with inclusion constraints.
\end{abstract}

Key words. Stability theory, containment problem, set-valued mappings, semi-infinite systems.

AMS subject classification. 49K40, 90C25, 90C 34

1. Introduction. The set containment problem consists of deciding, given two systems in $\mathbb{R}^{n}, y_{0}$ and $z_{0}$, whether the corresponding solution sets, $F_{0}$ and $G_{0}$, satisfy $F_{0} \subset G_{0}$ or not. A practical application of this set containment problem is the design centering problem which considers a container set $C \subset \mathbb{R}^{n}$ and a parametrized body $B(\lambda) \subset \mathbb{R}^{n}$, with parameter $\lambda \in \Lambda$. The idea is to maximize some functional (e.g. the volume of $B(\lambda)$ ) on the set $\{\lambda \in \Lambda: B(\lambda) \subset C\}$ (see [13]); the problem of cutting a diamond with accepted form and maximal volume from a raw diamond is an example. The multi-body design centering problem consider $B(\lambda)$ to be a finite union of nonoverlapping bodies (with finitely many connected components). It is also possible to consider the question about if, given $\bar{\lambda} \in \Lambda$ such that $B(\bar{\lambda}) \subset C$, then $B(\lambda) \subset C$ for $\lambda$ close enough to $\bar{\lambda}$. When the sets $C$ and $B(\lambda)$ are described by inequality constraints

$$
C=\left\{x \in \mathbb{R}^{n}: c_{s}(x) \leq 0, s \in S\right\}
$$

and

$$
B(\lambda)=\left\{x \in \mathbb{R}^{n}: b_{j}(x) \leq 0, j \in J ; \quad \omega_{\lambda}(x) \leq 0\right\}
$$

for some sets of indexes $S$ and $J$, while $\lambda \in \Lambda$, then one can pose the problem of studying the stability of the inclusion $B(\lambda) \subset C$ when some of the data $\left(c_{s}, b_{j}, \omega_{\lambda}\right)$ are subject to small perturbations.

Another application is the knowledge-based data classification problem: $F_{0}$ represents the knowledge set (which is fixed) whereas $G_{0}$ belongs to a predetermined family of sets from which the decision maker selects one of the best for a certain optimality criterion under the condition that $F_{0} \subset G_{0}$. This problem was posed in [4] (where $F_{0}$ is a given convex polyhedron and $G_{0}$ is required to be a half-space) and extended by Mangasarian, Jeyakumar et al. to more general situations: in [10] and [11], where $F_{0}$ and $G_{0}$ are solution sets of ordinary systems of differentiable constraints; in [8], which deals with ordinary convex systems and linear semi-infinite systems (in

\footnotetext{
${ }^{*}$ Corresponding author. Dep. of Statistics and Operations Research, Universidad de Alicante, Ctra. San Vicente de Raspeig s/n, 03071 Alicante, Spain. E-Mail: mgoberna@ua.es. Research supported by MCYT of Spain and FEDER of EU, Grant MTM2005-08572-C03-01

${ }^{\dagger}$ Faculty of Economics, Universidad Nacional de Cuyo, 5500 Mendoza, Argentina. E-Mail: vvera@fcemail.uncu.edu.ar. Research supported by SECYT-UNCuyo of Argentina, Grant 658/05-R
} 
brief, LSISs) and, finally, in [5], where also convex semi-infinite systems (CSISs) are considered. The last paper considers the stable containment problem for LSISs, i.e., deciding whether the inclusion of solution sets of such kind of systems is preserved by sufficiently small perturbation of the coefficients.

This paper extends the stable containment problem to CSISs, to general semiinfinite systems and even to arbitrary set-valued mappings.

More in detail, we consider given two set-valued mappings $\mathcal{F}: Y \rightrightarrows \mathbb{R}^{n}$ and $\mathcal{G}: Z \rightrightarrows \mathbb{R}^{n}$, where $\left(Y, \rho_{Y}\right)$ and $\left(Z, \rho_{Z}\right)$ are pseudometric spaces and a couple $\left(y_{0}, z_{0}\right) \in$ $Y \times Z$.

We say that the containment $\mathcal{F}\left(y_{0}\right) \subset \mathcal{G}\left(z_{0}\right)$ is stable (in brief, $\mathcal{F} \subset \mathcal{G}$ stably) at $\left(y_{0}, z_{0}\right)$ if there exists $\varepsilon>0$ such that $\mathcal{F}(y) \subset \mathcal{G}(z)$ for all $(y, z) \in Y \times Z$ with $\rho_{Y}\left(y, y_{0}\right)<\varepsilon$ and $\rho_{Z}\left(z, z_{0}\right)<\varepsilon$.

In the case of systems, we consider given $y_{0}=\left\{f_{t}^{0}(x) \leq 0, t \in T\right\}$ and $z_{0}=$ $\left\{g_{s}^{0}(x) \leq 0, s \in S\right\}$, where the index sets $T$ and $S$ are arbitrary and $f_{t}^{0}, g_{s}^{0}: \mathbb{R}^{n} \rightarrow \mathbb{R}$ are bounded functions on bounded sets, for all $t \in T$ and for all $s \in S$. We denote by $Y$ the class of all systems of the form $y=\left\{f_{t}(x) \leq 0, t \in T\right\}$ (i.e., those systems which have the same space of variables, $\mathbb{R}^{n}$, and the same index set, $T$ ) and, in a similar way, the space of parameters associated with $z_{0}$, say $Z$. These spaces are to be considered equipped with certain pseudometrics which provide the uniform convergence on the closed balls centered at the origin. Here $\mathcal{F}$ and $\mathcal{G}$ will be the feasible set mappings, i.e., $\mathcal{F}(y)$ and $\mathcal{G}(z)$ are the solution sets of $y=\left\{f_{t}(x) \leq 0, t \in T\right\}$ and $z=\left\{g_{t}(x) \leq 0, s \in S\right\}$, respectively.

We recall the stability concepts and some basic results for set-valued mappings that we shall consider in this paper. Let $\mathcal{F}: Y \rightrightarrows \mathbb{R}^{n}$ be a set-valued mapping. Its domain is $\operatorname{dom} \mathcal{F}:=\{y \in Y: \mathcal{F}(y) \neq \emptyset\}$. The following semicontinuity concepts are due to Bouligand and Kuratowski (see [1, Section 1.4]).

We say that $\mathcal{F}$ is lower semicontinuous at $y_{0} \in Y$ (lsc, in brief) if, for each open set $W \subset \mathbb{R}^{n}$ such that $W \cap \mathcal{F}\left(y_{0}\right) \neq \emptyset$, there exists an open set $V \subset Y$, containing $y_{0}$, such that $W \cap \mathcal{F}(y) \neq \emptyset$ for each $y \in V$. Obviously, $\mathcal{F}$ is lsc at $y_{0} \notin \operatorname{dom} \mathcal{F}$ and $y_{0} \in \operatorname{int} \operatorname{dom} \mathcal{F}$ if $\mathcal{F}$ is lsc at $y_{0} \in \operatorname{dom} \mathcal{F}$.

$\mathcal{F}$ is upper semicontinuous at $y_{0} \in Y$ (usc, in brief) if, for each open set $W \subset \mathbb{R}^{n}$ such that $\mathcal{F}\left(y_{0}\right) \subset W$, there exists an open set $V \subset Y$, containing $y_{0}$, such that $\mathcal{F}(y) \subset W$ for each $y \in V$. Clearly, if $\mathcal{F}$ is usc at $y_{0} \notin \operatorname{dom} \mathcal{F}$, then $y_{0} \in \operatorname{int}(Y \backslash \operatorname{dom} \mathcal{F})$.

If $\mathcal{F}$ is simultaneously lsc and usc at $y_{0}$ we say that $\mathcal{F}$ is continuous at this point.

$\mathcal{F}$ is closed at $y_{0} \in \operatorname{dom} \mathcal{F}$ if for all sequences $\left\{y_{r}\right\} \subset Y$ and $\left\{x_{r}\right\} \subset \mathbb{R}^{n}$ satisfying $x_{r} \in \mathcal{F}\left(y_{r}\right)$ for all $r \in \mathbb{N}, y_{r} \rightarrow y_{0}$ and $x_{r} \rightarrow x_{0}$, one has $x_{0} \in \mathcal{F}\left(y_{0}\right)$. If $\mathcal{F}$ is usc at $y_{0} \in \operatorname{dom} \mathcal{F}$ and $\mathcal{F}\left(y_{0}\right)$ is closed, then $\mathcal{F}$ is closed at $y_{0}$. Conversely, if $\mathcal{F}$ is closed and locally bounded at $y_{0} \in \operatorname{dom} \mathcal{F}$ (i.e., if there are a neighborhood of $y_{0}$, say $V$, and a bounded set $A \subset \mathbb{R}^{n}$ containing $\mathcal{F}(y)$ for every $\left.y \in V\right)$, then $\mathcal{F}$ is usc at $y_{0}$.

$\mathcal{F}$ is lsc (usc, closed, locally bounded) if it is lsc (usc, closed, locally bounded) at $y$ for all $y \in Y$.

The boundary mapping of $\mathcal{F}$ is bd $\mathcal{F}: Y \rightrightarrows \mathbb{R}^{n}$ such that $($ bd $\mathcal{F})(y)=\operatorname{bd} \mathcal{F}(y)$ for all $y \in Y$. In [7] has been shown that bd $\mathcal{F}$ is usc at $y_{0}$ if $\mathcal{F}$ is usc at $y_{0}$ and $\mathcal{F}(y)$ is the convex hull of bd $\mathcal{F}(y)$ for $y$ close to $y_{0}$.

Other notions of lower and upper semicontinuity as lsc and usc in the sense of Hausdorff (see, e.g., [2]) or inner and outer semicontinuity (see, e.g., [12], where it is shown that the last two concepts are equivalent to lsc and closedness when $\mathcal{F}$ is closed-valued) will not be considered in particular in this paper.

As an illustrative example, consider two particular instances of the design center- 
ing problem:

(a) Determining the greatest closed ball contained in $C$. Here $Y=\mathbb{R}^{3} \times \mathbb{R}_{++}$and, given $y=\left(y_{1}, y_{2}\right) \in Y, y_{1}$ represents the center of the ball and $y_{2}$ its radius.

(b) Determining the greatest object of a prescribed form (fixed by means of some compact pattern set) contained in $C$. Now the decision variables are the position of the gravity center (translation), the orientation of the selected axis (rotation), and the scale factor (dilation), so that $Y \subset \mathbb{R}^{7}$.

In both cases $\mathcal{G}$ is the constant mapping $\mathcal{G}(z)=C$ (for any pseudometric space $Z$ ) and both set-valued mappings, $\mathcal{F}$ and $\mathcal{G}$, are obviously lsc, usc, and closed.

Finally, some additional notation: the Euclidean and the Tchebyshev norms in $\mathbb{R}^{n}$ are denoted by $\|\cdot\|$ and $\|\cdot\|_{\infty}$, respectively. The Euclidean open ball centered at $x$ and radius $r>0$ is represented by $B(x ; r) .0_{n}$ is the null vector in $\mathbb{R}^{n}$. For any subsets $A, B \subset \mathbb{R}^{n}, d(A, B)$ denotes $\inf \{\|x-y\|: x \in A, y \in B\}$, with $\inf \emptyset=+\infty$. If $A$ is a subset of $\mathbb{R}^{n}, \operatorname{bd} A$, int $A$, and $\operatorname{cl} A$ represent the boundary, the interior, and the closure of $A$, respectively.

The paper is organized as follows. Section 2 deals with arbitrary set-valued mappings, including conditions for $\mathcal{F} \subset \mathcal{G}$ stably at $\left(y_{0}, z_{0}\right)$, which involve semicontinuity properties of $\mathcal{F}$ at $y_{0}$ and $\mathcal{G}$ at $z_{0}$, together with geometric conditions like $\mathcal{F}\left(y_{0}\right) \subset \operatorname{int} \mathcal{G}\left(z_{0}\right)$ or $d\left[\mathcal{F}\left(y_{0}\right)\right.$, bd $\left.\mathcal{G}\left(z_{0}\right)\right]>0$. Taking into account the generality of the set-valued mappings considered in this section, it is not surprising that we need to impose several hypotheses in order to get some sufficient or necessary conditions for the stability of the set containment. Section 3 exploits the results developed in Section 2 for obtaining analogous properties for semi-infinite systems. Since this section considers specially structured set-valued mappings by focussing the attention on the solution sets of CSISs and LSISs, the hypotheses discussed in the previous section are now quite easy to state.

2. Set-valued mappings. Throughout this section we assume that $\mathcal{F}: Y \rightrightarrows$ $\mathbb{R}^{n}$ and $\mathcal{G}: Z \rightrightarrows \mathbb{R}^{n}$ are two given set-valued mappings, with $\left(Y, \rho_{Y}\right)$ and $\left(Z, \rho_{Z}\right)$ pseudometric spaces, and $\left(y_{0}, z_{0}\right) \in Y \times Z$.

We show in this section that, under suitable assumptions, $d\left[\mathcal{F}\left(y_{0}\right)\right.$, bd $\left.\mathcal{G}\left(z_{0}\right)\right]>0$ implies that $\mathcal{F} \subset \mathcal{G}$ stably at $\left(y_{0}, z_{0}\right)$ whereas this property entails $\mathcal{F}\left(y_{0}\right) \subset \operatorname{int} \mathcal{G}\left(z_{0}\right)$.

Lemma 2.1. Let $\left(y_{0}, z_{0}\right) \in Y \times Z$ such that $\mathcal{F}\left(y_{0}\right) \subset \mathcal{G}\left(z_{0}\right)$ and there exists an open connected set $U$ such that $\mathcal{F}\left(y_{0}\right) \subset U \subset \mathcal{G}\left(z_{0}\right)$. Then

(i) $d\left[U, \operatorname{bd} \mathcal{G}\left(z_{0}\right)\right]>0$,

(ii) $\mathcal{F}$ is usc at $y_{0}$,

(iii) $\mathcal{G}$ is lsc at $z_{0}$, and

(iv) bd $\mathcal{G}$ is usc at $z_{0}$,

imply that $\mathcal{F} \subset \mathcal{G}$ stably at $\left(y_{0}, z_{0}\right)$.

Proof. If $\mathcal{F}\left(y_{0}\right)=\emptyset$, by (ii) we have $\mathcal{F}(y)=\emptyset$ in a certain neighborhood of $y_{0}$. Thus we assume that $\mathcal{F}\left(y_{0}\right) \neq \emptyset$. If $\mathcal{G}\left(z_{0}\right)=\mathbb{R}^{n}$ and so bd $\mathcal{G}\left(z_{0}\right)=\emptyset$, by (iii) and (iv) $\mathcal{G}(z)=\mathbb{R}^{n}$ for $z$ close enough to $z_{0}$. In the following discussion we confine ourselves to the non-trivial cases in which $\mathcal{F}\left(y_{0}\right) \neq \emptyset$ and $\mathcal{G}\left(z_{0}\right) \neq \mathbb{R}^{n}$.

Let $V:=\mathbb{R}^{n} \backslash \operatorname{cl} U$. Obviously, $U \cap V=\emptyset$ and bd $\mathcal{G}\left(z_{0}\right) \subset V$ by (i).

Since $\mathcal{F}\left(y_{0}\right) \subset U$, by (ii), there exists $\varepsilon_{1}>0$ such that

$$
\mathcal{F}(y) \subset U \text { if } \rho_{Y}\left(y, y_{0}\right)<\varepsilon_{1} .
$$


As $\emptyset \neq \mathcal{F}\left(y_{0}\right) \subset \mathcal{G}\left(z_{0}\right) \cap U$ and $\mathcal{G}$ is lsc at $z_{0}$, there exists $\varepsilon_{2}>0$ such that

$$
\mathcal{G}(z) \cap U \neq \emptyset \text { if } \rho_{Z}\left(z, z_{0}\right)<\varepsilon_{2} .
$$

Because bd $\mathcal{G}\left(z_{0}\right) \subset V$ and (iv) holds, there exists $\varepsilon_{3}>0$ such that

$$
\operatorname{bd} \mathcal{G}(z) \subset V \text { if } \rho_{Z}\left(z, z_{0}\right)<\varepsilon_{3} .
$$

Finally we prove that $\mathcal{F}(y) \subset \mathcal{G}(z)$ if $\rho_{Y}\left(y, y_{0}\right)<\varepsilon$ and $\rho_{Z}\left(z, z_{0}\right)<\varepsilon$, for $\varepsilon:=\min \left\{\varepsilon_{1}, \varepsilon_{2}, \varepsilon_{3}\right\}$. By $(2.1)$, it is enough to show that $U \subset \mathcal{G}(z)$ for all $z \in Z$ such that $\rho_{Z}\left(z, z_{0}\right)<\varepsilon$.

Let us assume the contrary, i.e., $U \nsubseteq \mathcal{G}(z)$. Take $x^{1} \in U \backslash \mathcal{G}(z)$. By $(2.2)$, we can take also $x^{2} \in \mathcal{G}(z) \cap U$. Since $U$ is an open connected set, there exists a continuous mapping $\alpha:[0,1] \rightarrow U$ such that $\alpha(0)=x^{1}$ and $\alpha(1)=x^{2}$. Let

$$
\bar{t}:=\sup \{t \in[0,1]: \alpha(t) \in \mathcal{G}(z)\} .
$$

Then $x^{3}:=\alpha(\bar{t}) \in[\operatorname{bd} \mathcal{G}(z)] \backslash V$ because $U \cap V=\emptyset$, in contradiction with (2.3).

Theorem 2.2. Let $\left(y_{0}, z_{0}\right) \in Y \times Z$ such that $\mathcal{F}\left(y_{0}\right) \subset \mathcal{G}\left(z_{0}\right)$ and

(i) $d\left[\mathcal{F}\left(y_{0}\right), \operatorname{bd} \mathcal{G}\left(z_{0}\right)\right]>0$,

(ii) $\mathcal{F}$ is usc at $y_{0}$,

(iii) $\mathcal{G}$ is lsc at $z_{0}$, and

(iv) bd $\mathcal{G}$ is usc at $z_{0}$.

Then, any of the following three conditions guarantees that $\mathcal{F} \subset \mathcal{G}$ stably at $\left(y_{0}, z_{0}\right)$ :

(a) $\mathcal{F}\left(y_{0}\right)$ is bounded.

(b) $\mathcal{F}\left(y_{0}\right)$ has finitely many connected components.

(c) $\mathcal{G}\left(z_{0}\right)$ is a convex set.

Proof. We assume $\mathcal{F}\left(y_{0}\right) \neq \emptyset$. Take $\mu:=\frac{1}{2} d\left[\mathcal{F}\left(y_{0}\right)\right.$, bd $\left.\mathcal{G}\left(z_{0}\right)\right]$.

First suppose that $\mathcal{F}\left(y_{0}\right)$ is bounded. Consider the open bounded sets

$$
U_{1}:=\mathcal{F}\left(y_{0}\right)+B\left(0_{n} ; \mu / 2\right) \subset U_{2}:=\mathcal{F}\left(y_{0}\right)+B\left(0_{n} ; \mu\right) \subset \operatorname{int} \mathcal{G}\left(z_{0}\right) .
$$

Since $\mathcal{F}\left(y_{0}\right) \subset U_{1}$ and bd $\mathcal{G}\left(z_{0}\right) \subset V:=\mathbb{R}^{n} \backslash \operatorname{cl} U_{2}$, there exists $\varepsilon>0$ such that

$$
\mathcal{F}(y) \subset U_{1} \text { if } \rho_{Y}\left(y, y_{0}\right)<\varepsilon
$$

and

$$
\operatorname{bd} \mathcal{G}(z) \subset V \text { if } \rho_{Z}\left(z, z_{0}\right)<\varepsilon .
$$

If $U_{1} \subset \mathcal{G}(z)$ for all $z$ close enough to $z_{0}$, then we are done. Otherwise, there exists a sequence $\left\{z_{r}\right\}$ such that $z_{r} \rightarrow z_{0}$ and $U_{1} \nsubseteq \mathscr{G}\left(z_{r}\right)$ for all $r=1,2, \ldots$. Take $x^{r} \in$ $U_{1} \backslash \mathcal{G}\left(z_{r}\right)$ and assume without loss of generality that $x^{r} \rightarrow \bar{x}$ Then $\bar{x} \in \operatorname{cl} U_{1} \subset U_{2}$ and we may consider some open ball $B$ centered at $\bar{x}$ contained in $U_{2}$. Since $B \cap \mathcal{G}\left(z_{0}\right) \neq \emptyset$, condition (iii) implies that $B \cap \mathcal{G}\left(z_{r}\right) \neq \emptyset$ for $r$ large enough. Let $r_{0}$ be such that $x^{r} \in B, B \cap \mathcal{G}\left(z_{r}\right) \neq \emptyset$ and bd $\mathcal{G}\left(z_{r}\right) \subset V$ for all $r \geq r_{0}$. The fact that $B$ is connected yields a contradiction as in the proof of the previous lemma.

Second assume that $\mathcal{F}\left(y_{0}\right)$ is connected. The set $U:=\mathcal{F}\left(y_{0}\right)+B\left(0_{n} ; \mu\right)$ is open and connected. Since $d\left[U, \operatorname{bd} \mathcal{G}\left(z_{0}\right)\right]=\mu>0$, Lemma 2.1 applies.

In the case that $\mathcal{F}\left(y_{0}\right)$ has finitely many connected components, say $F_{i}, i=$ $1, \ldots, k$, we only need to replace $\varepsilon_{2}$ in the proof of the previous lemma by $\varepsilon_{2}=$ 
$\min _{i=1, \ldots, k} \eta_{i}$, where each $\eta_{i}>0$ is such that $\mathcal{G}(z) \cap\left(F_{i}+B\left(0_{n} ; \mu\right)\right) \neq \emptyset$ if $\rho_{Z}\left(z, z_{0}\right)<$ $\eta_{i}$ and consider an appropriate connected component.

Finally we assume that $\mathcal{G}\left(z_{0}\right)$ is a convex set. The set

$$
U:=\left\{x \in \operatorname{int} \mathcal{G}\left(z_{0}\right): d\left[x, \operatorname{bd} \mathcal{G}\left(z_{0}\right)\right]>\mu\right\}
$$

is open and satisfies $\mathcal{F}\left(y_{0}\right) \subset U$. Now we prove that $U$ is convex. Let $x^{i} \in U, i=1,2$. Since int $\mathcal{G}\left(z_{0}\right)$ is convex and $B\left(x^{i} ; \mu\right) \subset \operatorname{int} \mathcal{G}\left(z_{0}\right), i=1,2$, we have

$$
\bigcup_{\lambda \in[0,1]}\left\{(1-\lambda) B\left(x^{1} ; \mu\right)+\lambda B\left(x^{2} ; \mu\right)\right\} \subset \operatorname{int} \mathcal{G}\left(z_{0}\right),
$$

so that $d\left[(1-\lambda) x^{1}+\lambda x^{2}\right.$, bd $\left.\mathcal{G}\left(z_{0}\right)\right]>\mu$ for all $\lambda \in[0,1]$. Hence $\left[x^{1}, x^{2}\right] \subset U$ and Lemma 2.1 applies again.

Corollary 2.3. Let $\mathcal{G}$ be closed-convex-valued and let $\left(y_{0}, z_{0}\right) \in Y \times Z$. If $\mathcal{F}\left(y_{0}\right)$ is a closed convex subset of $\operatorname{int} \mathcal{G}\left(z_{0}\right), \mathcal{G}\left(z_{0}\right)$ is bounded, $\mathcal{F}$ is usc at $y_{0}$ and $\mathcal{G}$ is continuous at $z_{0}$, then $\mathcal{F} \subset \mathcal{G}$ stably at $\left(y_{0}, z_{0}\right)$.

Proof. Under the assumptions $\mathcal{G}$ is locally bounded at $z_{0}$, so that the convex hull of bd $\mathcal{G}$ coincides with $\mathcal{G}$ in a certain neighborhood of $z_{0}$. Thus bd $\mathcal{G}$ is continuous. On the other hand, since $\mathcal{F}\left(y_{0}\right)$ and bd $\mathcal{G}\left(z_{0}\right)$ are disjoint compact sets, $d\left[\mathcal{F}\left(y_{0}\right)\right.$, bd $\left.\mathcal{G}\left(z_{0}\right)\right]>$ 0. $\mathrm{c}$

EXAMPLE 2.4. The next four cases show that none of the four conditions (i)-(iv) in Theorem 2.2 is superfluous. In all of them $\mathcal{F}$ and $\mathcal{G}$ are solution sets mappings corresponding to linear systems in $\mathbb{R}^{2}$ (so that they are closed-convex-valued). The sets $Y$ and $Z$ are endowed with the usual topology in $\mathbb{R}$.

(a) Let $Y=Z=\mathbb{R}_{++}$,

$$
\mathcal{F}(y)=\left\{x \in \mathbb{R}^{2}:-y \leq x_{i} \leq y, i=1,2\right\},
$$

$\mathcal{G}=\mathcal{F}$ and $y_{0}=z_{0}=1$. $\mathcal{F}$ (and so $\mathcal{G}$ ) is continuous, so that (ii)-(iv) hold. Nevertheless $\mathcal{F}(y) \nsubseteq \mathcal{G}(z)$ if $z<1<y$.

(b) Let $Y=Z=\mathbb{R}_{+}$,

$$
\begin{gathered}
\mathcal{F}(y)=\left\{x \in \mathbb{R}^{2}: x_{1}+y x_{2} \geq 0,-x_{1}+y x_{2} \geq 0\right\}, \\
\mathcal{G}(z)=\left\{x \in \mathbb{R}^{2}:-1 \leq x_{1} \leq 1\right\}
\end{gathered}
$$

(constant) and $y_{0}=z_{0}=0$. Now (i), (iii) and (iv) hold whereas $\mathcal{F}(y) \nsubseteq \mathcal{G}\left(z_{0}\right)$ if $y>0$.

(c) Let $Y=Z=\mathbb{R}$,

$$
\mathcal{F}(y)=\left\{x \in \mathbb{R}^{2}:-1 \leq x_{i} \leq 1, i=1,2\right\}
$$

(constant),

$$
\mathcal{G}(z)=\left\{x \in \mathbb{R}^{2}:-2 \leq x_{i} \leq 2, i=1,2 ; 0_{2}^{\prime} x \leq z\right\}
$$

and $y_{0}=z_{0}=0$. Obviously, (i), (ii) and (iv) hold but $\mathcal{F}(0)=[-1,1]^{2} \nsubseteq \mathcal{G}(z)=\emptyset$ if $z<0$.

(d) Let $Y=Z=[0,1], \mathcal{F}(y)=\left\{x \in \mathbb{R}^{2}: x_{i} \geq 1, i=1,2\right\}$ (constant),

$$
\mathcal{G}(z)=\left\{x \in \mathbb{R}^{2}:-x_{1}+z x_{2} \leq 0, z x_{1}-x_{2} \leq 0\right\}
$$


and $y_{0}=z_{0}=0$. It is easy to see that (i)-(iii) hold but $\mathcal{F}\left(y_{0}\right) \nsubseteq \mathcal{G}(z)$ if $0<z<1$.

It is an open problem to know whether the alternative conditions (a), (b), (c) in Theorem 2.2 are superfluous or not.

In [6] it is shown the existence of consistent LSISs such that the solution set is constant under arbitrary but sufficiently small perturbation of the coefficients (i.e., $\mathcal{F}$ is constant in a certain neighborhood of $\left.y_{0}\right)$, so we can have $\mathcal{F} \subset \mathcal{G}$ stably at $\left(y_{0}, z_{0}\right)$ and nevertheless $\mathcal{F}\left(y_{0}\right) \nsubseteq \operatorname{int} \mathcal{G}\left(z_{0}\right)$ (take for instance $\mathcal{G}=\mathcal{F}$ ). The next result provides conditions guaranteeing that the inclusion $\mathcal{F}\left(y_{0}\right) \subset \operatorname{int} \mathcal{G}\left(z_{0}\right)$ is necessary for the stability of $\mathcal{F} \subset \mathcal{G}$ at $\left(y_{0}, z_{0}\right)$. The intuitive meaning of such conditions are that $\mathcal{F}$ expands in the proximity of $y_{0}$ and $\mathcal{G}$ shrinks inwards close to $z_{0}$, respectively.

Condition A: $\mathcal{F}$ satisfies that for all closed half-space $S$ such that $\mathcal{F}\left(y_{0}\right) \subset S$ and bd $S$ supports $\mathcal{F}\left(y_{0}\right)$, there exists a sequence $\left\{y_{r}\right\} \subset Y$ such that $y_{r} \rightarrow y_{0}$ and

$$
\mathcal{F}\left(y_{r}\right) \backslash S \neq \emptyset \text { for all } r \in \mathbb{N} \text {. }
$$

Condition B: $\mathcal{G}$ satisfies that for all supporting hyperplanes to $\mathcal{G}\left(z_{0}\right)$, say $H$, there exists $\left\{z_{r}\right\} \subset Z$ such that $z_{r} \rightarrow z_{0}$ and

$$
\mathcal{G}\left(z_{r}\right) \cap H=\emptyset \text { for all } r \in \mathbb{N} \text {. }
$$

Observe that if $y_{0}=\left\{f_{t}^{0}(x) \leq 0, t \in T\right\}$ is a system such that $\mathcal{F}\left(y_{0}\right)$ is convex and the family of function constraints, $\left\{f_{t}^{0}, t \in T\right\}$, is equilipschitzian, then $\mathcal{F}$ satisfies the condition A. Indeed, if $S$ is any closed half-space $\omega^{\prime}(x-u) \leq 0$ such that $\|\omega\|=1$, $u \in \operatorname{bd} \mathcal{F}\left(y_{0}\right)$ and $\mathcal{F}\left(y_{0}\right) \subset S$, the sequence $\left\{y_{r}\right\} \subset Y$,

$$
y_{r}:=\left\{f_{t}^{0}\left(x-\frac{\omega}{r}\right) \leq 0, t \in T\right\}, r \in \mathbb{N},
$$

verifies that $y_{r} \rightarrow y_{0}$ and

$$
\mathcal{F}\left(y_{r}\right) \backslash S \neq \emptyset \text { for all } r \in \mathbb{N},
$$

because $x^{r}:=u+\frac{\omega}{r} \in \mathcal{F}\left(y_{r}\right)$ and

$$
\omega^{\prime}\left(x^{r}-u\right)=\omega^{\prime}\left(u+\frac{\omega}{r}-u\right)=\frac{1}{r}\|\omega\|^{2}=\frac{1}{r}>0,
$$

so $x^{r} \notin S$.

The family of the function constraints of a convex system $\left\{f_{t}^{0}(x) \leq 0, t \in T\right\}$ is equilipschitzian whenever the set of all the corresponding subgradients is bounded because, if $M>0$ satisfies that $\cup_{t \in T} \partial f_{t}^{0}\left(\mathbb{R}^{n}\right) \subset B\left(0_{n} ; M\right)$, then the inequality

$$
f_{t}^{0}\left(x^{1}\right)-f_{t}^{0}\left(x^{2}\right) \geq u_{t}^{\prime}\left(x^{1}-x^{2}\right) \geq-M\left\|x^{1}-x^{2}\right\|,
$$

for any $x^{1}, x^{2} \in \mathbb{R}^{n}$ (here $u_{t}$ is a subgradient of $f_{t}^{0}$ at $x^{2}$ ), yields that

$$
\left|f_{t}^{0}\left(x^{1}\right)-f_{t}^{0}\left(x^{2}\right)\right| \leq M\left\|x^{1}-x^{2}\right\| .
$$


Similarly, consider an equilipschitzian family $z_{0}=\left\{g_{t}^{0}, s \in S\right\}$ such that $\mathcal{G}\left(z_{0}\right)$ is convex. Let $H$ be any supporting hyperplane to $\mathcal{G}\left(z_{0}\right)$. Let $\omega^{\prime}(x-v)=0$ be the equation of $H$, with $\|\omega\|=1, v \in$ bd $\mathcal{G}\left(z_{0}\right)$ and $\omega^{\prime}(x-v) \leq 0$ for all $x \in \mathcal{G}\left(z_{0}\right)$. Then the sequence $\left\{z_{r}\right\} \subset Z$,

$$
z_{r}:=\left\{g_{s}^{0}\left(x+\frac{\omega}{r}\right) \leq 0, s \in S\right\}, r \in \mathbb{N},
$$

satisfies $z_{r} \rightarrow z_{0}$ and $\mathcal{G}\left(z_{0}\right)=\frac{\omega}{r}+\mathcal{G}\left(z_{r}\right)$. Thus

$$
x \in \mathcal{G}\left(z_{r}\right) \Rightarrow x+\frac{\omega}{r} \in \mathcal{G}\left(z_{0}\right),
$$

which implies that $\omega^{\prime}\left(x+\frac{\omega}{r}-v\right) \leq 0$, so that

$$
\omega^{\prime}(x-v) \leq-\frac{1}{r}\|\omega\|^{2}<0
$$

Hence

$$
\mathcal{G}\left(z_{r}\right) \cap H=\emptyset \text { for all } r \in \mathbb{N} .
$$

Therefore we have shown that $\mathcal{G}$ satisfies condition $\mathbf{B}$.

THEOREM 2.5. Let $\mathcal{F} \subset \mathcal{G}$ stably at $\left(y_{0}, z_{0}\right)$ and assume that $\mathcal{G}\left(z_{0}\right)$ is convex and either $\mathcal{F}$ satisfies condition $\boldsymbol{A}$ or $\mathcal{G}$ satisfies condition $\boldsymbol{B}$. Then $\mathcal{F}\left(y_{0}\right) \subset \operatorname{int} \mathcal{G}\left(z_{0}\right)$.

Proof. We assume that $\mathcal{F} \subset \mathcal{G}$ stably at $\left(y_{0}, z_{0}\right)$ but $\mathcal{F}\left(y_{0}\right) \not \subset \operatorname{int} \mathcal{G}\left(z_{0}\right)$. Let $\bar{x} \in \mathcal{F}\left(y_{0}\right) \backslash \operatorname{int} \mathcal{G}\left(z_{0}\right)$. Then $\bar{x} \in \operatorname{bd} \mathcal{G}\left(z_{0}\right)$, and so by the supporting hyperplane theorem there exists $w \in \mathbb{R}^{n} \backslash\left\{0_{n}\right\}$ such that

$$
w^{\prime}(x-\bar{x}) \leq 0 \text { for all } x \in \operatorname{cl} \mathcal{G}\left(z_{0}\right) .
$$

Since $\bar{x} \in \mathcal{F}\left(y_{0}\right) \subset \mathcal{G}\left(z_{0}\right)$, the hyperplane

$$
H:=\left\{x \in \mathbb{R}^{n}: w^{\prime}(x-\bar{x})=0\right\}
$$

also supports $\mathcal{F}\left(y_{0}\right)$ at $\bar{x}$. Put

$$
S:=\left\{x \in \mathbb{R}^{n}: w^{\prime}(x-\bar{x}) \leq 0\right\} .
$$

We will get a contradiction from both conditions $\mathbf{A}$ and $\mathbf{B}$. If $\mathbf{A}$ holds, we can take $\left\{y_{r}\right\} \subset Y, y_{r} \rightarrow y_{0}, \mathcal{F}\left(y_{r}\right) \backslash S \neq \emptyset$ for all $r \in \mathbb{N}$. Since $\mathcal{G}\left(z_{0}\right) \subset S$, we also have $\mathcal{F}\left(y_{r}\right) \backslash \mathcal{G}\left(z_{0}\right) \neq \emptyset$, so that $\mathcal{F}\left(y_{r}\right) \not \subset \mathcal{G}\left(z_{0}\right)$ for all $r$.

Alternatively, if $\mathbf{B}$ holds there exists $\left\{z_{r}\right\} \subset Z, z_{r} \rightarrow z_{0}$, such that $\mathcal{G}\left(z_{r}\right) \cap H=\emptyset$ for all $r$. Since $\bar{x} \in H$, we have $\bar{x} \notin \mathcal{G}\left(z_{r}\right)$, so that $\bar{x} \in \mathcal{F}\left(y_{0}\right) \backslash \mathcal{G}\left(z_{r}\right)$ and we have $\mathcal{F}\left(y_{0}\right) \not \subset \mathcal{G}\left(z_{r}\right)$ for all $r$.

In both cases we get a contradiction.

The conclusion $\mathcal{F}\left(y_{0}\right) \subset \operatorname{int} \mathcal{G}\left(z_{0}\right)$ in the previous theorem cannot be replaced by the stronger one $d\left(\mathcal{F}\left(y_{0}\right)\right.$, bd $\left.\mathcal{G}\left(z_{0}\right)\right)>0$, as the set-valued mappings $\mathcal{F}$ and $\mathcal{G}$ (both of them solution set mappings corresponding to parametric systems) in the following examples show. Nonetheless, in the next section we prove this property for semi-infinite systems under mild assumptions. 
Example 2.6. Let $Y=Z=] 0,1]$,

$$
\mathcal{F}(y)=\left\{x \in \mathbb{R}^{2}: x_{1} \geq 0, x_{2} \geq 0, x_{1} x_{2} \geq y\right\},
$$

$\mathcal{G}(z)=\left\{x \in \mathbb{R}^{2}: x_{1} \geq 0, x_{2} \geq 0\right\}$ (constant) and $y_{0}=z_{0}=1$. Then $\mathcal{F} \subset \mathcal{G}$ stably at $\left(y_{0}, z_{0}\right)$, condition $\boldsymbol{A}$ is satisfied and $\mathcal{F}\left(y_{0}\right) \subset \operatorname{int} \mathcal{G}\left(z_{0}\right)$ but $d\left(\mathcal{F}\left(y_{0}\right), \operatorname{bd} \mathcal{G}\left(z_{0}\right)\right)=0$.

Example 2.7. Let $Y=Z=[0,1[$,

$$
\mathcal{F}(y)=\left\{x \in \mathbb{R}^{2}: x_{1} \geq 0, x_{2} \geq 0, x_{1} x_{2} \geq 1\right\}
$$

(constant),

$$
\mathcal{G}(z)=\left\{x \in \mathbb{R}^{2}: x_{1} \geq 0, x_{2} \geq 0, x_{1} x_{2} \geq z\right\}
$$

and $y_{0}=z_{0}=0$. Then $\mathcal{F} \subset \mathcal{G}$ stably at $\left(y_{0}, z_{0}\right)$, condition $\boldsymbol{B}$ holds and $\mathcal{F}\left(y_{0}\right) \subset$ $\operatorname{int} \mathcal{G}\left(z_{0}\right)$, but once again $d\left(\mathcal{F}\left(y_{0}\right), \operatorname{bd} \mathcal{G}\left(z_{0}\right)\right)=0$.

3. Solution sets of systems. Throughout this section we consider two given (nominal) systems $y_{0}=\left\{f_{t}^{0}(x) \leq 0, t \in T\right\}$ and $z_{0}=\left\{g_{t}^{0}(x) \leq 0, s \in S\right\}$ with corresponding spaces of parameters, denoted by $Y$ and $Z$ in the general case, by $Y_{C}$ and $Z_{C}$ if $y_{0}$ and $z_{0}$ are convex systems and by $Y_{L}$ and $Z_{L}$ if $y_{0}$ and $z_{0}$ are linear systems. $\mathcal{F}$ and $\mathcal{G}$ are the feasible set mappings, i.e., $\mathcal{F}(y)$ and $\mathcal{G}(z)$ are the solution sets of $y=\left\{f_{t}(x) \leq 0, t \in T\right\}$ and $z=\left\{g_{t}(x) \leq 0, s \in S\right\}$, respectively.

In the case of linear systems $y_{0}=\left\{\left(a_{t}^{0}\right)^{\prime} x \leq b_{t}^{0}, t \in T\right\}$ and $z_{0}=\left\{\left(c_{s}^{0}\right)^{\prime} x \leq d_{s}^{0}\right.$, $s \in S\}$, where the index sets $T$ and $S$ are arbitrary and $a_{t}^{0}, c_{s}^{0} \in \mathbb{R}^{n}, b_{t}^{0}, d_{s}^{0} \in \mathbb{R}$ for all $t \in T$ and for all $s \in S$. We denote by $Y_{L}$ the class of all the linear systems of the form $y=\left\{\left(a_{t}\right)^{\prime} x \leq b_{t}, t \in T\right\}$, (i.e., those linear systems which have the same space of variables, $\mathbb{R}^{n}$, and the same index set, $T$ ). We consider in $Y_{L}$ the topology of the uniform convergence on $T$ given by the pseudometric

$$
\rho_{Y_{L}}\left(y_{1}, y_{2}\right):=\sup _{t \in T}\left\|\left(\begin{array}{c}
a_{t}^{1} \\
b_{t}^{1}
\end{array}\right)-\left(\begin{array}{c}
a_{t}^{2} \\
b_{t}^{2}
\end{array}\right)\right\|_{\infty},
$$

for $y_{i}=\left\{\left(a_{t}^{i}\right)^{\prime} x \leq b_{t}^{i}, t \in T\right\} \in Y_{L}, i=1,2$.

If $y_{0}=\left\{f_{t}^{0}(x) \leq 0, t \in T\right\}$ is convex, we associate with $y_{0}$ the space $Y_{C}$ of all the convex systems of the form $y=\left\{f_{t}(x) \leq 0, t \in T\right\}$, with $f_{t}: \mathbb{R}^{n} \rightarrow \mathbb{R}$ convex for all $t \in$ $T$. As in [9], we define a pseudometric $\rho_{Y_{C}}$ as follows: given $y_{i}=\left\{f_{t}^{i}(x) \leq 0, t \in T\right\} \in$ $Y_{C}, i=1,2$,

$$
\rho_{Y_{C}}\left(y_{1}, y_{2}\right):=\sup _{t \in T} \delta\left(f_{t}^{1}, f_{t}^{2}\right)
$$

where

$$
\delta\left(f_{t}^{1}, f_{t}^{2}\right):=\sum_{k=1}^{\infty} 2^{-k} \frac{\delta_{k}\left(f_{t}^{1}, f_{t}^{2}\right)}{1+\delta_{k}\left(f_{t}^{1}, f_{t}^{2}\right)}
$$

with 


$$
\delta_{k}\left(f_{t}^{1}, f_{t}^{2}\right):=\sup _{\|x\| \leq k}\left|f_{t}^{1}(x)-f_{t}^{2}(x)\right|, k=1,2, \ldots .
$$

If a sequence $\left\{y_{r}\right\} \subset Y_{C}$ satisfies $y_{r} \rightarrow y_{0}$, with $y_{r}=\left\{f_{t}^{r}(x) \leq 0, t \in T\right\} \in Y_{C}$, $r=1,2, \ldots$, then for all $x \in \mathbb{R}^{n}, f^{r}(x) \rightarrow f^{0}(x)$ uniformly on $T$. Moreover $f_{t}^{r} \rightarrow f_{t}^{0}$ uniformly over compact sets in $\mathbb{R}^{n}$ because of the convexity of the functions $f_{t}^{r}$ and $f_{t}^{0}$. It is easy to prove that the topology induced by $\rho_{Y_{C}}$ on $Y_{L}$ coincides with the topology associated with the pseudometric of the uniform convergence on $T$ given by $d_{Y_{L}}$.

In the general case, $y_{0}=\left\{f_{t}^{0}(x) \leq 0, t \in T\right\}$, the index set $T$ is arbitrary and the functions to be considered, $f_{t}^{0}: \mathbb{R}^{n} \rightarrow \mathbb{R}$, are bounded over bounded sets (not necessarily convex functions). We associate with $y_{0}$ the space $Y$ of all the systems of the form $y=\left\{f_{t}(x) \leq 0, t \in T\right\}$, where $f_{t}: \mathbb{R}^{n} \rightarrow \mathbb{R}$ is any function bounded over bounded sets, for each $t \in T$. Finally, we consider in $Y$ the topology induced by the pseudometric $\rho_{Y}$ defined in the same way as for the space $Y_{C}$, see (3.1).

The topology of $Y_{L}$ is the topology induced $Y_{C}$ and the topology of $Y_{C}$ is the one induced by $Y$. These facts yield that all the sufficient conditions for the stability properties of multivalued mappings defined on $Y_{C}(Y)$ are inherited by their restrictions to $Y_{L}\left(Y_{C}\right)$. Nonetheless, the necessary conditions may require a direct argument. We represent by $\mathcal{F}$ the feasible set mapping for $Y_{L}$ or $Y_{C}$ or $Y$ in either case.

In a similar way, the space of parameters associated with $z_{0}, Z_{L}$ or $Z_{C}$ or $Z$, is equipped with the pseudometric $d_{Z_{L}}$ or $\rho_{Z_{C}}$ or $\rho_{Z}$, respectively.

First we will start with the general case. For non-convex systems it is not possible to relax the assumptions in Theorem 2.2. To get some necessary conditions we could have applied Theorem 2.5, but we prefer for practical uses to replace assumptions $\mathbf{A}$ and $\mathbf{B}$ by some conditions easier to verify, getting the stronger necessary condition $d\left(\mathcal{F}\left(y_{0}\right)\right.$, bd $\left.\mathcal{G}\left(z_{0}\right)\right)>0$, which is not true in general as Examples 2.6 and 2.7 show.

TheOREM 3.1. Let $\left(y_{0}, z_{0}\right) \in Y \times Z$ be such that $\mathcal{G}\left(z_{0}\right)$ is convex and at least one of the two families of function constraints, $\left\{f_{t}^{0}, t \in T\right\}$ and $\left\{g_{s}^{0}, s \in S\right\}$, is equilipschitzian. If $\mathcal{F} \subset \mathcal{G}$ stably at $\left(y_{0}, z_{0}\right)$, then $d\left(\mathcal{F}\left(y_{0}\right)\right.$, bd $\left.\mathcal{G}\left(z_{0}\right)\right)>0$ (and so, $\left.\mathcal{F}\left(y_{0}\right) \subset \operatorname{int} \mathcal{G}\left(z_{0}\right)\right)$.

Proof. Assume that there exists $M>0$ such that

$$
\left|f_{t}^{0}\left(x^{1}\right)-f_{t}^{0}\left(x^{2}\right)\right| \leq M\left\|x^{1}-x^{2}\right\|,
$$

for all $x^{1}, x^{2} \in \mathbb{R}^{n}$. Take $\delta>0$ such that $\mathcal{F}(y) \subset \mathcal{G}(z)$ for any $(y, z) \in Y \times Z$ with $\rho_{Y}\left(y, y_{0}\right)<\delta$ and $\rho_{Z}\left(z, z_{0}\right)<\delta$. We will use the fact (proved below, at the end) that if $w \in \mathbb{R}^{n}$ and $y=\left\{f_{t}^{0}(x-w) \leq 0, t \in T\right\}$ then

$$
\rho_{Y}\left(y, y_{0}\right) \leq \frac{M\|w\|}{1+M\|w\|} .
$$

In particular, for any $\omega \in \mathbb{R}^{n},\|\omega\|=1$, if $y_{r}:=\left\{f_{t}^{0}\left(x-\frac{\omega}{r}\right) \leq 0, t \in T\right\}, r \in \mathbb{N}$, we get

$$
\rho_{Y}\left(y_{r}, y_{0}\right) \leq \frac{M\left\|\frac{\omega}{r}\right\|}{1+M\left\|\frac{\omega}{r}\right\|}=\frac{M}{r+M}
$$


Take $r_{0} \in \mathbb{N}$ such that $\frac{M}{r_{0}+M}<\delta$ and let $\varepsilon=\frac{1}{2 r_{0}}>\varepsilon$. Suppose that the distance $d\left(\mathcal{F}\left(y_{0}\right), \operatorname{bd} \mathcal{G}\left(z_{0}\right)\right)=0$. Consider $u \in \mathcal{F}\left(y_{0}\right)$ and $v \in \operatorname{bd} \mathcal{G}\left(z_{0}\right)$ such that $\|u-v\|<$ $\varepsilon$. By the supporting hyperplane theorem we can choose some $\omega \in \mathbb{R}^{n},\|\omega\|=1$, such that

$$
\omega^{\prime}(x-v) \leq 0 \text { for all } x \in \mathcal{G}\left(z_{0}\right) .
$$

For $y_{r_{0}}$ we have that $\rho_{Y}\left(y_{r_{0}}, y_{0}\right)<\delta$, and so $\mathcal{F}\left(y_{r_{0}}\right) \subset \mathcal{G}\left(z_{0}\right)$ by the choice of $\delta$. Since $\mathcal{F}\left(y_{r_{0}}\right)=\mathcal{F}\left(y_{0}\right)+\frac{\omega}{r_{0}}$ and $u \in \mathcal{F}\left(y_{0}\right)$, it follows that $u+\frac{\omega}{r_{0}} \in \mathcal{G}\left(z_{0}\right)$. Then, by (3.3),

$$
0 \geq \omega^{\prime}\left(u+\frac{\omega}{r_{0}}-v\right)=\frac{1}{r_{0}}\|\omega\|^{2}+\omega^{\prime}(u-v) \geq \frac{1}{r_{0}}-\|u-v\|>\frac{1}{r_{0}}-\varepsilon=\frac{1}{2 r_{0}}>0,
$$

a contradiction. Therefore it must be that $d\left(\mathcal{F}\left(y_{0}\right)\right.$, bd $\left.\mathcal{G}\left(z_{0}\right)\right)>0$.

In case the equilipschitzian family is the one corresponding to the mapping $\mathcal{G}$, the result follows in the same fashion as above by considering the sequence given by $z_{r}:=\left\{g_{s}^{0}\left(x+\frac{\omega}{r}\right) \leq 0, s \in S\right\}$.

To finish the proof we only need to show the assertion (3.2). For any $k \in \mathbb{N}$,

$$
\delta_{k}\left(f_{t}^{0}(\cdot), f_{t}^{0}(\cdot-w)\right):=\max _{\|x\| \leq k}\left|f_{t}^{0}(x)-f_{t}^{0}(x-w)\right| \leq M\|w\| .
$$

Since the function $s \mapsto \frac{s}{1+s}$ is increasing on $\mathbb{R}_{+}$, for any $t \in T$ we have

$$
\begin{aligned}
\delta\left(f_{t}^{0}(\cdot), f_{t}^{0}(\cdot-w)\right) & :=\sum_{k=1}^{\infty} 2^{-k} \frac{\delta_{k}\left(f_{t}^{0}(\cdot), f_{t}^{0}(\cdot-w)\right)}{1+\delta_{k}\left(f_{t}^{0}(\cdot), f_{t}^{0}(\cdot-w)\right)} \\
& \leq \sum_{k=1}^{\infty} 2^{-k} \frac{M\|w\|}{1+M\|w\|} \\
& =\frac{M\|w\|}{1+M\|w\|},
\end{aligned}
$$

so that

$$
\rho_{Y}\left(y, y_{0}\right):=\sup _{t \in T} \delta\left(f_{t}^{0}(\cdot), f_{t}^{0}(\cdot-w)\right) \leq \frac{M\|w\|}{1+M\|w\|} .
$$

This completes the proof.

Theorem 3.2. Let $y_{0}$ and $z_{0}$ be convex systems such that $\mathcal{F}\left(y_{0}\right) \neq \emptyset$. Then the following statements hold:

(i) If $\mathcal{F}\left(y_{0}\right) \subset \operatorname{int} \mathcal{G}\left(z_{0}\right), \mathcal{G}$ is lsc at $z_{0}$, and $\mathcal{G}\left(z_{0}\right)$ is bounded, then $\mathcal{F} \subset \mathcal{G}$ stably at $\left(y_{0}, z_{0}\right)$.

(ii) If $\mathcal{F} \subset \mathcal{G}$ stably at $\left(y_{0}, z_{0}\right)$ and the set of subgradients of the function constraints is bounded for at least one of them, then $d\left(\mathcal{F}\left(y_{0}\right)\right.$, bd $\left.\mathcal{G}\left(z_{0}\right)\right)>0$ and $\mathcal{G}$ is lsc at $z_{0}$.

Proof. (i) Since $\mathcal{F}\left(y_{0}\right)$ and $\mathcal{G}\left(z_{0}\right)$ are bounded, $\mathcal{F}$ and $\mathcal{G}$ are usc at $y_{0}$ and $z_{0}$ respectively. The conclusion follows from Corollary 2.3.

(ii) The argument is the same as in the previous theorem.

Different characterizations of the lsc property of $\mathcal{G}$ at $z_{0}$ that can be checked in terms of the data have been given in [9]. For ordinary (finite) systems the boundedness condition in (ii) can be replaced by the lipschitzian property of the function constraints of one of the involved systems. 
The event of the family of the function constraints of the linear system $\left\{a_{t}^{\prime} x \leq b_{t}\right.$, $t \in T\}$ is even easier because

$$
\left\|\left(a_{t}^{\prime} x^{1}-b_{t}\right)-\left(a_{t}^{\prime} x^{2}-b_{t}\right)\right\| \leq\left\|a_{t}\right\|\left\|x^{1}-x^{2}\right\| \leq M\left\|x^{1}-x^{2}\right\|,
$$

if $\left\|a_{t}\right\| \leq M$ for all $t \in T$. Hence, together with Corollary 2.3, we get the following result for linear systems ([5]):

Theorem 3.3. Let $y_{0}$ and $z_{0}$ be linear systems such that $\mathcal{F}\left(y_{0}\right) \neq \emptyset$. Then the following statements hold:

(i) If $\mathcal{F}\left(y_{0}\right) \subset \operatorname{int} \mathcal{G}\left(z_{0}\right), \mathcal{G}$ is lsc at $z_{0}$ and $\mathcal{G}\left(z_{0}\right)$ is bounded, then $\mathcal{F} \subset \mathcal{G}$ stably at $\left(y_{0}, z_{0}\right)$.

(ii) If $\mathcal{F} \subset \mathcal{G}$ stably at $\left(y_{0}, z_{0}\right)$ and the set of gradients of either $y_{0}$ or $z_{0}$ is bounded, then $\mathcal{F}\left(y_{0}\right) \subset \operatorname{int} \mathcal{G}\left(z_{0}\right)$ and $\mathcal{G}$ is lsc at $z_{0}$.

In LSISs, the boundedness of $\mathcal{G}\left(z_{0}\right)$ in (i) can be replaced by the weaker characterization of the usc property of $\mathcal{G}$ that can be found in [3], with the inconvenient that this property is not inherited by $\mathcal{F}$ and, moreover, it can hardly be checked in practice. In the particular case that $z_{0}$ is an ordinary linear systems, $\mathcal{G}$ is lsc at $z_{0}$ if and only if Slater condition holds and it usc at $z_{0}$ if and only if $\mathcal{G}\left(z_{0}\right)$ is either bounded or the whole space $\mathbb{R}^{n}$.

Corollary 3.4. Let $y_{0}$ and $z_{0}$ be ordinary linear systems such that $\mathcal{F}\left(y_{0}\right) \neq \emptyset$. Then the following statements hold:

(i) If $\mathcal{F}\left(y_{0}\right) \subset \operatorname{int} \mathcal{G}\left(z_{0}\right)$ and $\mathcal{G}$ is continuous at $z_{0}$, then $\mathcal{F} \subset \mathcal{G}$ stably at $\left(y_{0}, z_{0}\right)$.

(ii) If $\mathcal{F} \subset \mathcal{G}$ stably at $\left(y_{0}, z_{0}\right)$, then $\mathcal{F}\left(y_{0}\right) \subset \operatorname{int} \mathcal{G}\left(z_{0}\right)$ and $\mathcal{G}$ is lsc at $z_{0}$.

We have assumed in this section that $y_{0}$ and $z_{0}$ are systems of the same class. However, analogous results for mixed combinations can be obtained in a similar way.

\section{REFERENCES}

[1] Aubin, J.-P., Frankowska, H.: Set-Valued Analysis. Birkhäuser-Verlag, Boston (1990)

[2] Bank, B., Guddat, J., Klatte, D., Kummer, B., Tammer, K.: Non-Linear Parametric Optimization. Birkhäuser-Verlag, Basel (1983)

[3] Cánovas, M.J., López, M.A., Parra, J.: Upper semicontinuity of the feasible set mapping for linear inequality systems. Set-Valued Analysis 10, 361-378 (2002)

[4] Fung, G., Mangasarian, O.L., Shavlik, J.: Knowledge-based support vector machine classifiers. In: Becker, S., Thrun, S., Obermayer, K. (eds.) Neural Information Processing Systems, pp. 521-528. MIT Press, Cambridge, MA (2003)

[5] Goberna, M.A., Jeyakumar, V., Dinh, N.: Dual characterizations of set containments with strict convex inequalities. J. Global Optim. 34, 33-54 (2006)

[6] Goberna, M.A., Larriqueta, M., Vera de Serio, V.N.: On the stability of the boundary of the feasible set in linear optimization. Set-valued Anal. 11, 203-223 (2003)

[7] Goberna, M.A., López, M.A., Todorov, M.I.: On the stability of closed-convex-valued mappings and the associated boundaries. J. Math. Anal. Appl. 306, 502-515 (2005)

[8] Jeyakumar, V.: Characterizing set containments involving infinite convex constraints and reverse-convex constraints. SIAM J. Optim. 13, 947-959 (2003)

[9] Lopez, M.A., Vera de Serio, V.N.: Stability of the feasible set mapping in convex semi-infinite programming. In: Goberna, M.A., López, M.A. (eds.) Semi-Infinite Programming: Recent Advances, pp. 101-120, Kluwer, Dordrecht (2001)

[10] Mangasarian, O.L.: Mathematical programming in data mining. Data Mining and Knowledge Discovery 1, 183-201 (1997) 
[11] Mangasarian, O.L.: Set Containment characterization. J. Global Optim. 24, 473-480 (2002)

[12] Rockafellar, R.T., Wets, R.B.: Variational Analysis. Springer-Verlag, New York (1998).

[13] Stein, O.: A semi-infinite approach to design centering. In: Dempe, S., Kalashnikov, S. (eds.) Optimization with Multivalued Mappings: Theory, Applications and Algorithms, Kluwer, Dordrecht, to appear. 